\title{
Improved Long Period Fibre Gratings sensing devices coated with thin films
}

\author{
L. Coelho \\ CAP / INESC TEC - Technology and Science and Faculty \\ of Sciences, University of Porto, Rua do Campo Alegre \\ Porto, Portugal \\ lcoelho@inesporto.pt
}

\author{
J. L. Santos \\ CAP / INESC TEC - Technology and Science and Faculty \\ of Sciences, University of Porto, Rua do Campo Alegre \\ Porto, Portugal \\ Department of Physics and Astronomy of Faculty of \\ Sciences, University of Porto \\ Porto, Portugal \\ jsantos@fc.up.pt
}

\author{
J. M. M. M. de Almeida \\ CAP / INESC TEC - Technology and Science and Faculty \\ of Sciences, University of Porto, Rua do Campo Alegre \\ Porto, Portugal \\ Department of Physics, School of Sciences and Technology, \\ Universidade de Trás-os-Montes e Alto Douro \\ Vila Real, Portugal \\ immma@utad.pt \\ P.A.S. Jorge \\ CAP / INESC TEC - Technology and Science and Faculty \\ of Sciences, University of Porto, Rua do Campo Alegre \\ Porto, Portugal \\ Department of Physics and Astronomy of Faculty of \\ Sciences, University of Porto \\ Porto, Portugal \\ pedro.jorge@.fc.up.pt
}

\begin{abstract}
Long period fibre gratings (LPFG) have found an increasing interest due to the easy fabrication in any kind of fibre through the induced electric arc technique with low cost and flexibility. They have been used in applications such as gainflattening and band-rejection, and for sensing strain, temperature and chemical and biological parameters. The sensitivity of LPFG increases when the fibre is coated with certain thin films. In this short review it is presented the recent developments and applications of these kind of sensors.
\end{abstract}

Keywords - Long period gratings, Thin film coating, Fibre optic sensor, Fibre optic refractometer.

\section{INTRODUCTION}

Over the past thirty years, many efforts were focused on sensing devices based on small fibre optic structures because they have a wide range of interesting properties. They are simple and versatile, have small size and are flexible, possess high melting point, high sensitivity and bandwidth. In addition, they have the ability to monitor several parameters simultaneous and allow multiplexing. They can be used to measure several physical or chemical parameters but they are mostly used as refractometers for measuring refractive index (RI) variations. An extensive work has been done in this field using a wide variety of configurations with different type of structures. Fibres with inline interferometers, with gratings or even using the well-known plasmonic effect have returned high sensitivity measurement of the external RI [1].

The optical fibre gratings, also called band-rejection filters, are easy to produce with high reproducibility while maintaining robustness. They can be inscribed in common single-mode optical fibres by introducing a small modulation in the RI of the core. Varying the period of the modulation results in fibre Bragg gratings (FBG), with periods of few hundred nanometres), or in long period fibre gratings (LPFG), with periods from $100 \mu \mathrm{m}$ to $1 \mathrm{~mm}$, depending on the wavelength range of operation.

FBGs induce contra directional coupling. Light energy from forward propagating modes is transferred to modes traveling in the opposite direction. FBGs are often referred to as short period, reflection gratings because of this effect. When using FBGs the fundamental guided mode never leaves the core. The evanescent electromagnetic field only "sees" the external medium if the fibre cladding is removed either by polishing or by chemical etching, decreasing the robustness of the fibre.

LPFGs, in contrast, couple energy co-directionally. Energy from the forward propagating incident modes is transferred to either forward propagating cladding or radiation modes depending on the structure of the waveguide [2]. The light transmitted thought the LPFGs coupled to the cladding modes, result in several attenuation bands in the transmitted spectrum, where each one corresponds to a specific cladding mode [3, 4]. Since the evanescent field of those modes interact with the external medium they are intrinsically sensitive to the external RI without any post processing, therefore keeping the structure robust [5]. This feature allows the use of LPFGs as sensing element devices in chemical and biochemical analytical measurements [6].

LPFGs are potential candidates for optical fibre sensing devices. Precise and real time determination of the surrounding refractive index (SRI) using optical fibre sensors based on LPFGs is an objective of ongoing research, covering a wide range of applications. Their sensitivity can be enhanced greatly 
if the grating period, fibre dimensions and SRI are optimized in a way to operate at a point called turn around point on phase matching curves of these gratings. Sensors for sensing physical and chemical properties based on metal oxide coated LPFGs with improved wavelength and optical power sensitivities have been demonstrated recently.

\section{PRINCIPLE OF OPERATION}

Transmission spectrum of the LPFG consists of series of attenuation bands as shown in Fig. 1. The period of the RI modulation is such that a phase matching condition between the fundamental core mode and a forward propagating cladding mode of an optical fibre exists for a wavelength given by $[7,8]$ :

$$
\lambda=\left[n_{\text {eff }}(\lambda)-n_{\text {clad }}^{m}(\lambda)\right] \Lambda
$$

where $n_{\text {eff }}(\lambda)$ is the effective refractive index of the propagating core mode at wavelength $\lambda, n_{\text {clad }}^{m}(\lambda)$ is the effective RI of the $m^{\text {th }}$ cladding mode and $\Lambda$ is the period of the LPFG. As a result several attenuation bands are generated in the transmission spectrum of the fibre $[6,8]$. The period of the grating determines the wavelength of interaction and the strength of coupling is determined by the modal overlap given by the coupling coefficient. The minimum transmission of the attenuation bands is determined by the expression [6]:

$$
T_{i}=1-\sin ^{2}\left(k_{m} L\right)
$$

where $\mathrm{L}$ is the length of the LPFG and $\mathrm{k}_{\mathrm{m}}$ is the coupling coefficient for the $m^{\text {th }}$ cladding mode. The coupling coefficient is calculated by the overlap integral of core and cladding modes and amplitude of periodic modulation of the mode propagating constants:

$$
\kappa_{m}=\frac{\omega \varepsilon_{0}}{4} \int_{0}^{2 \pi} d \phi \int_{0}^{\infty} r d r \psi_{1}^{*} \Delta n \psi_{2}
$$

where $\psi_{1}$ and $\psi_{2}$ are the transverse field distributions of core and cladding modes and $\Delta \mathrm{n}$ is the peak change of the RI [6].

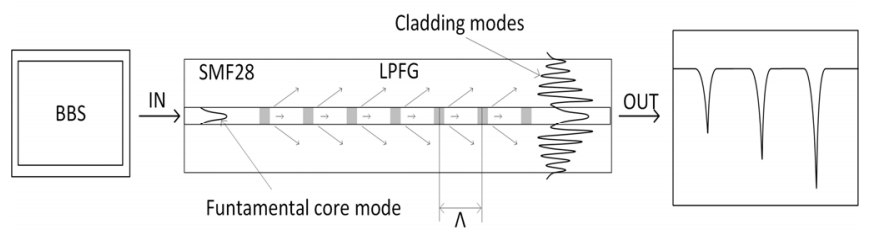

Fig. 1. Transmission spectrum of a broadband signal through an LPFG.

Efficient coupling exists when core and cladding modes have similar electric field profiles and, therefore, have a large overlap integral. Band position, their amplitudes and inter-band spaces depend on the fibre core and cladding diameters, RI difference, length of grating and modulation of the refractive index.

The high wavelength sensitivity of the LPFG spectrum to the SRI can be explained by the dependence of the phase matching condition on the effective RI of the cladding modes, which in turn depend on the difference between cladding and the surrounding medium [3].
Assuming that the LPFG period is unaffected under a change in SRI $\left(n_{s}\right)$ and that the effective RI of the core mode is unchanged, an expression can be obtained for the wavelength sensitivity of the $m^{\text {th }}$ cladding mode

$$
\left(\frac{d \lambda}{d n_{s}}\right)_{m}=\left(\frac{d \lambda}{d \delta n_{e f f, m}}\right)_{m}\left(\frac{d \delta n_{e f f, m}}{d n_{s}}\right)_{m}
$$

where

$$
\delta n_{e f f, m}=n_{e f f}(\lambda)-n_{\text {clad }}^{m}(\lambda)
$$

The term $\left(\frac{d \delta n_{e f f, m}}{d n_{s}}\right)_{m}$ is different for each cladding mode and therefore the sensitivity of an LPFG is dependent on the order of the cladding mode.

This sensitivity to the SRI can be used to design it as chemical sensors. As the SRI increases up to near the cladding refractive index (CRI), the principal effect is a shift to shorter wavelengths of the centre of the bands, particularly noticeable for the higher order modes [9].

It has been demonstrated [10] that for this kind of structures the highest wavelength and transmission sensitivity to the RI of the external medium occurs for SRI values slightly lower that the CRI [3]. Also, the sensitivity is higher for the higher order modes [11]. When the SRI is higher than the CRI all the attenuation bands show a considerable reduction in wavelength and transmission sensitivity [11].

Although the wavelength position of the LPFG attenuation band is highly dependent of the external medium, coating these modulated sections with high RI materials will change the coupling condition to the cladding mode [12, 13]. It was calculated and experimentally demonstrated that a high index layer induces strong changes on standard LPFG spectral properties [14].

The result is an improvement of the sensitivity allowing tuning of high sensitivity levels to a certain target. For instance, coating the LPFG with Langmuir-Blodgett nanolayers was first proposed by Tatam et al. [15]. Fig. 2 illustrates results presented by Coelho et al. on the enhancement of the sensitivity to the external medium of similar LPFGs coated with different oxides in a wide range of refractive indexes [16].

The wavelength position of the attenuation band corresponding to the $\mathrm{LP}_{1,6}$ cladding propagation mode changes with a nonlinear response following a tendency to increase the sensitivity when approaching the RI value of the fused silica. For higher values, the coated LPFGs have a very different behaviour and open the possibility to monitor high refractive index liquids, such as edible or mineral oils. 

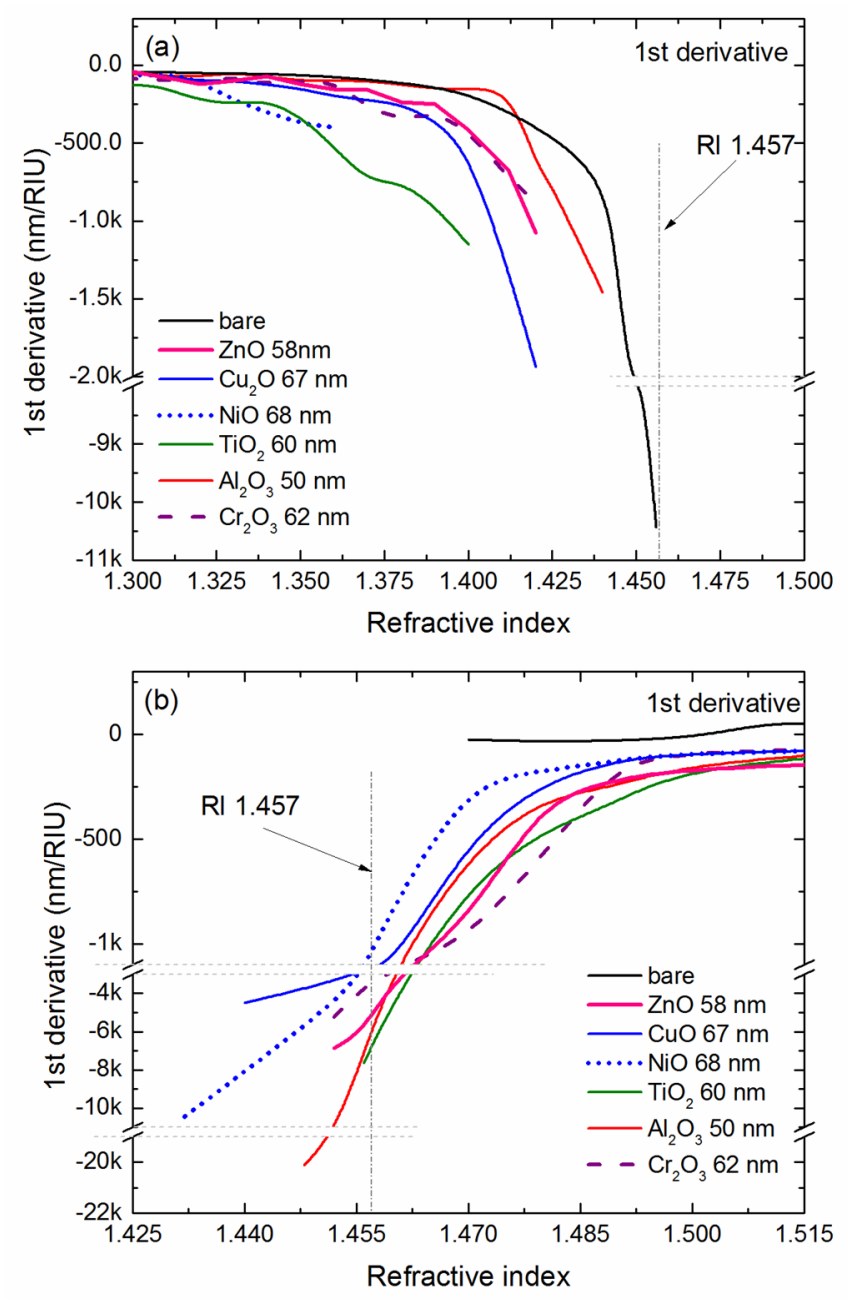

Fig. 2. Survey of the sensitivity to the external medium, below (a) and above (b) the CRI, of similar LPFGs coated with different oxides (from [16]).

\section{FABRICATION OF THIN FILM COATED LPFGS}

The most common techniques to obtain this modulation are UV laser irradiation, electric-arc discharge, periodic etching, $\mathrm{CO}_{2}$ laser irradiation and mechanical processes [9].

The fabrication of the LPFGs coated with thin film have been performed at INESC TEC where the induced electric arc technique was used to produce bare LPFGs [5]. They were formed in uncoated standard telecommunications single-mode fibres (SMF28, Corning, Inc.), by point-to-point short time electric discharges creating micro tapers. .A $10 \mathrm{~cm}$ long section of single-mode fibres was striped and cleaned and then placed between two electrodes $1 \mathrm{~mm}$ apart with one end clamped at a motorized linear stage and stretched in the other end with a mass of about $5 \mathrm{~g}$ to keep the tension constant. The electric arcs are produced by applying a current of $9 \mathrm{~mA}$ during $1 \mathrm{~s}$. After each electric arc the linear stage moves the fibre $387 \pm 0.1 \mu \mathrm{m}$, a value chosen to produce a resonance wavelength at $1.48 \mu \mathrm{m}$ matching the asymmetric $6^{\text {th }}$ order cladding mode. The process is repeated until the attenuation band reaches a value $>25 \mathrm{~dB}$ with final length typically of $45 \pm 5 \mathrm{~mm}$ [16].
The metal thin films were deposited around the grating region using an electron beam evaporator model Auto 306 (Edwards Ltd, U.K.). A thickness monitor model FTM5 (Edwards Ltd., U.K.) provide measurements with a resolution of $0.1 \mathrm{~nm}$. The adhesion between the fibre and the different metals was accomplished by a $3 \mathrm{~nm}$ buffer layer of $\mathrm{Cr}$. The equipment is fitted with a dedicated rotary system placed inside the chamber in order to rotate the fibres, as illustrated in Fig. 3. The rotation velocity was $5 \mathrm{rpm}$, a value found to produce homogeneous thin films around the fibres, therefore, variations in polarization should not return significant differences in the transmission sensitivity.

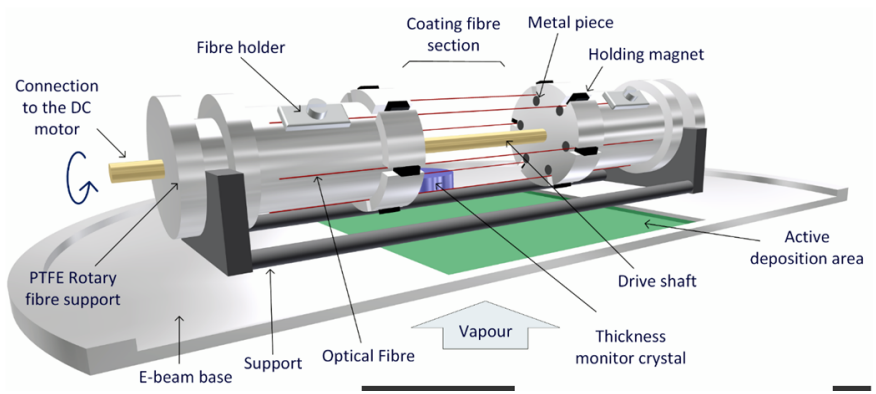

Fig. 3. Fabrication set-up placed inside the e-beam chamber in order to rotate the fibres to produce homogeneous thin films.

The thin film thickness of a set of static and rotating substrates was measured by the thickness monitor and confirmed with a surface profiler model DektakXT (Bruker Co., U.S.A.). With this data the real thickness of the metal deposited around the cylindrical fibre can be estimated.

In order to get dielectric thin films around the fibres, metal thin films with tenth of nanometers of thickness were first deposited around the LPFGs. They were then placed in a setup, illustrated in Fig. 4, where one end was clamped with a magnet and at the other end a $5 \mathrm{~g}$ weight kept the fibre stretched at a constant tension in the central region of a $520 \mathrm{~W}$ tubular furnace (Termolab, Portugal).

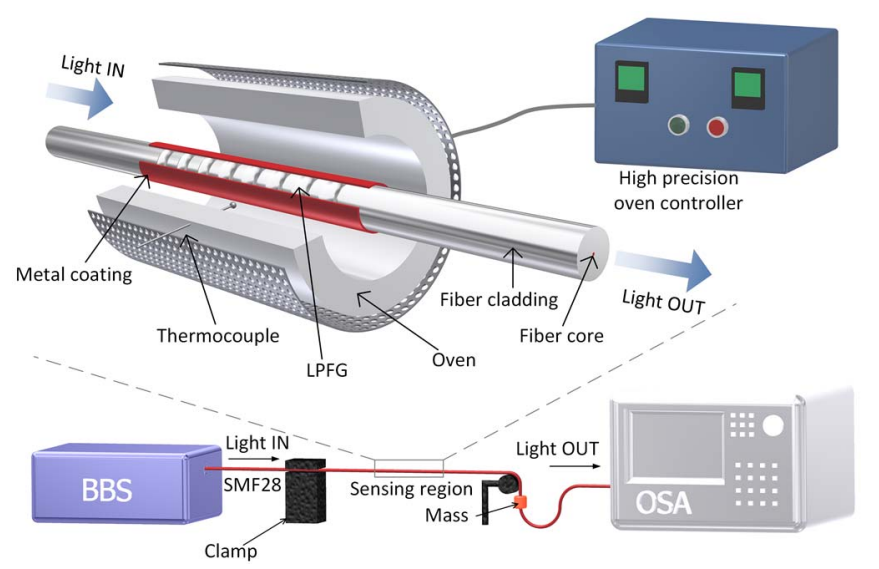

Fig. 4. Scheme of the oxidation and optical characterization setup using an oven and a LPFG working in transmission comprising a broadband source (BBS), an optical spectrum analyzer (OSA) and the grating sensor (from [16]). 
The temperature of the oven was risen up to $600^{\circ} \mathrm{C}$ at a rate of $\sim 5^{\circ} \mathrm{C}$ per minute and the cooling took place at approximately the same rate. The metal oxidation took place at normal atmospheric pressure and a temperature specific of each metal. Silicon substrates coated with patterned metal thin films with the same thickness were placed simultaneously in the furnace to be used in further morphologic analysis as the surface profiler in order to determine the final metal oxide thickness.

\section{APPLICATIONS}

Optical fibre sensors based on LPFGs have been widely studied for the last years for sensing physico-chemical properties of the external medium, such as strain, bending, temperature and refractive index [17-20]. They have been applied at INESC TEC for implementation of chemical sensors and biosensors based on the RI changes in the surrounding medium [5].

LPFGs are intrinsically sensitive to temperature, therefore, sensors based on these gratings have to be compensated for temperature fluctuations [17]. Sensors using the difference in measurand and temperature responses of FBGs and LPFGs to discriminate between the measurand and temperature induced wavelength shifts were proposed by Vengsarkar et al. [19] where sensor interrogation was performed entirely on the FBG reflection signals. Another approach for LPFG interrogation is based on a FBG/LPFG superstructure for temperature compensation, is illustrated in Fig. 5. The transmission spectrum of the sensor possesses several narrow-band loss peaks with intensity and wavelength dependences which, when combined with the information that can be retrieved from the FBG spectral signature, allows the simultaneous determination of strain and temperature [21].

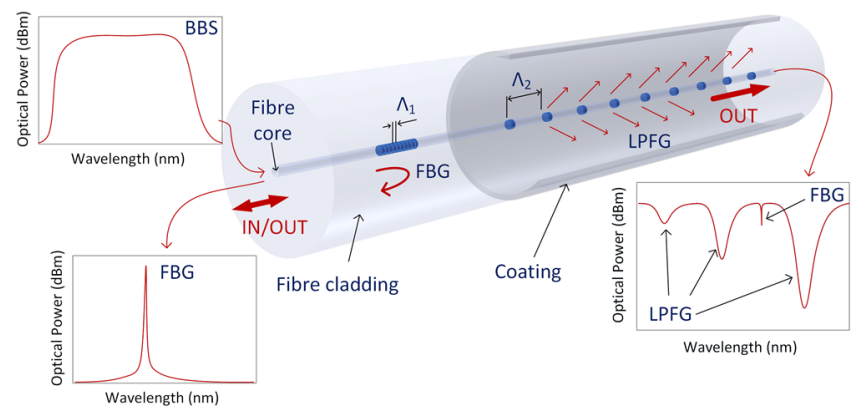

Fig. 5. Scheme of a fibre Bragg grating in-line with a long period fibre grating coated with a high refractive index material. The signal from the LPFG is monitored in transmission while the reflection from the FBG allows temperature monitoring.

Biosensors based on the RI changes have been developed at INESC TEC.

For example, the detection of thrombin based on aptamer binding is studied using two different optical fibre-based configurations illustrated in Fig. 6: long period gratings coated with a thin layer of titanium dioxide and surface plasmon resonance devices in optical fibres coated with a multilayer of gold and titanium dioxide. These structures are functionalized and the performance to detect thrombin in the range 10 to
$100 \mathrm{nM}$ is compared in transmission mode. The sensitivity to the SRI of the plasmonic device is higher than $3100 \mathrm{nmRIU}^{-1}$ in the RI range from 1.335 to 1.355 , a factor of 20 greater than the sensitivity of the coated grating. The detection of $10 \mathrm{nM}$ of thrombin was accomplished with a wavelength shift of $3.5 \mathrm{~nm}$ and a resolution of $0.54 \mathrm{nM}[22]$.
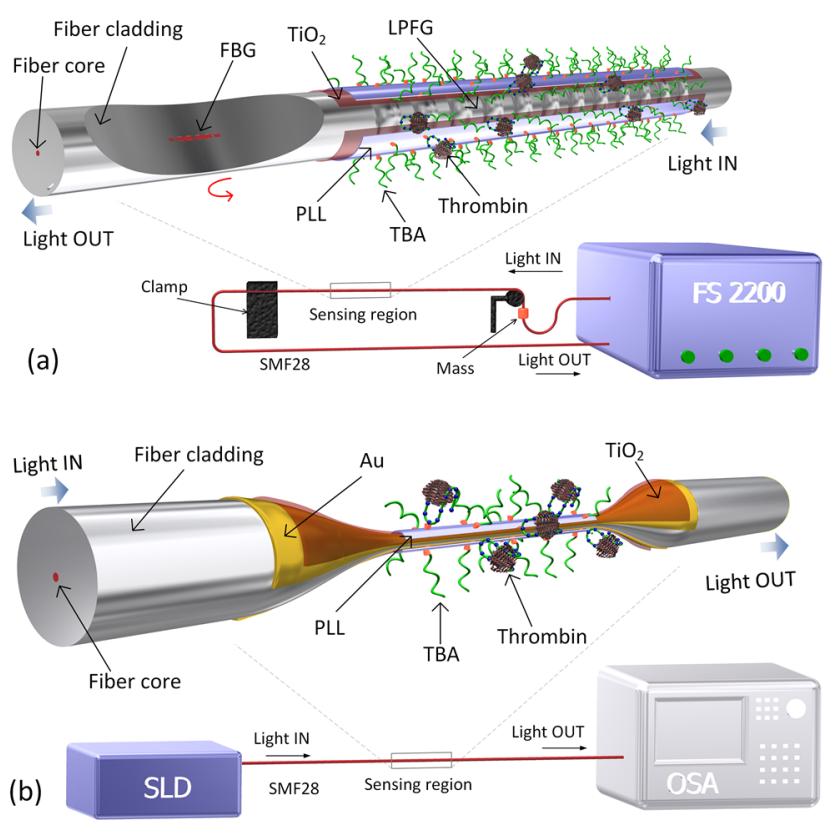

Fig. 6. Scheme of the sensing configurations: (a) LPFG coated with $30 \mathrm{~nm}$ of $\mathrm{TiO}_{2}$ and (b) surface plasmon resonance device in etched single-mode fibre coated with $\mathrm{Au}$ and $\mathrm{TiO}_{2}$ (from [22]).

In the food safety and public health area the detection of organic solvents (either in the liquid or in vapor phase) in foodstuff and monitoring thermal deterioration of edible oils can be emphasized.

An optical sensing scheme based on hybrid FBG combined with $\mathrm{TiO}_{2}$ coated LPFG for monitoring organic solvents in high RI edible oils was reported [23]. The readout of the sensor was implemented using either the wavelength shift of the LPFG resonance dip or the variation of the optical power level of the reflected/transmitted light at the FBG wavelength peak, which in turn depends on the wavelength position of the LPFG resonance. In order to investigate and optimize the sensor performance, two different FBG/LPFG interrogation systems were implemented. Hexane concentrations up to $20 \% \mathrm{~V} / \mathrm{V}$, corresponding to the 1.467 to $1.451 \mathrm{RI}$ range, were considered. For the transmission mode of operation, sensitivities of $1.41 \mathrm{~nm} / \% \mathrm{~V} / \mathrm{V}$ and $0.11 \mathrm{~dB} / \% \mathrm{~V} / \mathrm{V}$, with resolutions of 0.58 and $0.29 \% \mathrm{~V} / \mathrm{V}$, were achieved when using the LPFG wavelength shift and the FBG transmitted optical power, respectively. For the $\mathrm{FBG}$ reflection mode of operation, a lower sensitivity of $0.07 \mathrm{~dB} / \% \mathrm{~V} / \mathrm{V}$ and a resolution better than $0.16 \% \mathrm{~V} / \mathrm{V}$ were estimated.

Figure 7 illustrates the detection of volatile organic compounds accomplished with a sensing device based on a LPFG coated with a zinc oxide $(\mathrm{ZnO})$ thin layer with selftemperature compensation [24]. The $\mathrm{ZnO}$ coating structure was 
produced onto the cladding of the fibre by thermal oxidation of a metallic $\mathrm{Zn}$ thin film. The morphological characterization of $\mathrm{ZnO}$ thin films, grown at the same time on silicon substrates, was performed using X-ray diffraction, X-ray Photoelectron Spectroscopy and Scanning Electron Microscope which shows very good agreement. LPFGs with $290 \mathrm{~nm}$ thick $\mathrm{ZnO}$ coating were fabricated and characterized for the detection of ethanol and hexane in vapor phase. For ethanol a sensitivity of $0.99 \mathrm{~nm} / \mathrm{g} . \mathrm{m}^{-3}$ was achieved when using the wavelength shift interrogation mode, while for hexane a much lower sensitivity of $0.003 \mathrm{~nm} / \mathrm{g} \cdot \mathrm{m}^{-3}$ was measured, indicating a semi-selectivity of the sensor with a spectral resolution better than $3.2 \mathrm{~g} . \mathrm{m}^{-3}$.

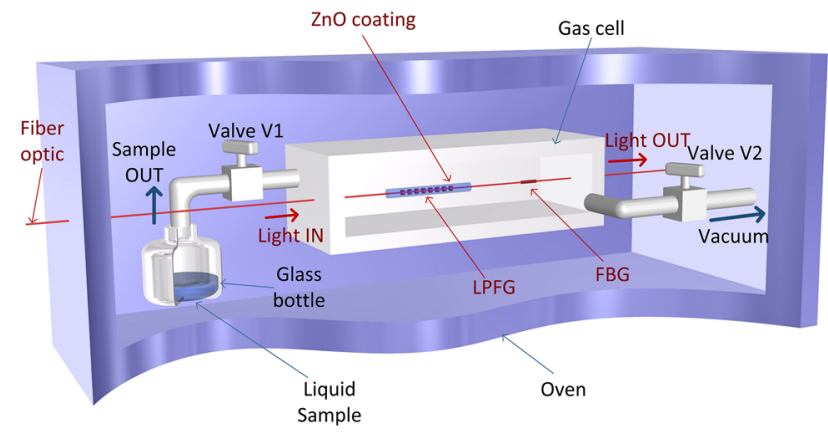

Fig. 7. Setup for the characterization of the sensor constituted by a glass bottle, valves and a gas cell placed inside an oven. A broadband source and an optical spectrum analyzer are not shown.

A new sensing system for the detection of thermal deterioration of extra virgin olive oil based on LPFG was reported. It was demonstrated the feasibility of LPFG sensor for the analysis of high RI edible oils [25]. The detection principle is the wavelength dependence of the attenuation bands of a $\mathrm{TiO}_{2}$ coated LPFG on the changes in the refractive index of the medium surrounding the cladding surface of the optical fibre. The quality of the sensor was tested by measuring the wavelength shift of the attenuation bands in response to thermal deterioration of an edible oil (extra virgin olive oil) with RI higher than the fibre cladding. Absorption spectroscopy allowed the effects of thermal deterioration to be detected, for example, in the decreasing of the absorption band at $677 \mathrm{~nm}$, attributed to chlorophyll A. A detection limit of about 5 minutes at $180^{\circ} \mathrm{C}$ and of about 2 minutes at $225^{\circ} \mathrm{C}$ was observed for the sensing system. The proposed sensing system could lead to the realization of a biochemical sensor for the food industry.

In the materials science area, phase transition of copper oxide were monitored using a copper coated LPFG. This study opens the possibility of monitoring chemical reactions and phase transitions in the more complex iron material system. LPFGs were used to monitor the characteristics of copper $(\mathrm{Cu})$ thin films when annealed in air atmosphere heated up to $\sim 680^{\circ} \mathrm{C}$ [26]. The wavelength and the optical power shift of the resonant bands of the LPFGs when coated with the $\mathrm{Cu}$ thin films, were measured as a function of the annealing temperature, and were found to exhibit a different evolution comparing to a bare LPFGs. Thin films of $\mathrm{Cu}$ deposited on quartz $\left(\mathrm{SiO}_{2}\right)$ substrates were annealed and analysed by XRD, SEM/EDS and Raman spectroscopy, allowing to identify the formation of two distinct oxide phases at different temperatures, cuprous ( $\mathrm{Cu} 2 \mathrm{O}$-cuprite) and cupric $(\mathrm{CuO}-$ tenorite) oxides, respectively. The observed features of the resonant bands of the LPFGs were found to be associated with the $\mathrm{Cu}$ oxide phase transitions, indicating the possibility of using LPFGs to monitor, in real time, the oxidation states of $\mathrm{Cu}$ thin films by following specific characteristics of the attenuation bands. In addition, LPFGs over coated with the two distinct oxidation phases of $\mathrm{Cu}$ were characterized for $\mathrm{RI}$ sensing in the range between 1.300 and 1.600 , leading to the conclusion that the sensitivity to the refractive index of the surrounding medium of $\mathrm{Cu}$ coated LPFGs sensing systems can be temperature tuned [26].

\section{CONCLUSION}

LPFGs coated with thin films, namely, metal oxide thin films are a promising platform for the implementation of fibre optic sensors. Applications of LPFGs in life sciences, environment monitoring and material sciences were already demonstrated. The dielectric coatings, as oxides, can be used to increase the sensitivity of bare LPFGs to the external refractive index mediums without losing the capabilities to be functionalized in order to the detection of specific targets as molecules or chemical entities. In addition, modifications in the coating structure can be monitored in real-time. The development of sensors for monitoring of corrosion in in-land and offshore metallic structure is being carried out at INESC TEC using this principle. It is important to point out that in spite of the existence of different techniques to produce different kind of LPFGs these type of coatings can be applied in a wide range of configurations maintaining the same physical properties. The absent of electrical devices makes these kind of structures ideal to be used in marine environments to monitor structural modifications of particular materials or the presence of certain chemical species in the water.

\section{ACKNOWLEDGMENT}

This work has received funding from the European Union's Horizon 2020 research and innovation programme under the project STRONGMAR: STRengthening MARitime technology Research Center (H202-TWINN-2015 (CSA)-692427). It was also financed by the "North Portugal Regional Operational Programme (NORTE 2020), under the PORTUGAL 2020 Partnership Agreement", and through the "European Regional Development Fund (ERDF)" within the project Coral Sustainable Ocean Exploitation: Tools and Sensors/NORTE01-0145-FEDER-000036.

\section{REFERENCES}

[1] P. A. S. Jorge et al., "Fiber Optic-Based Refractive Index Sensing at INESC Porto," Sensors, vol. 12, no. 6, pp. 8371-8389, JUN 20122012.

[2] J. F. Akki, A. S. Lalasangi, P. U. Raikar, T. Srinivas, L. S. Laxmeshwar, and U. Raikar, "Core-cladding mode resonances of long period fiber grating in concentration sensor," IOSR J. Appl. Phys, vol. 4, pp. 41-46, 2013. 
[3] H. Patrick, A. Kersey, and F. Bucholtz, "Analysis of the response of long period fiber gratings to external index of refraction," (in English), Journal of Lightwave Technology, Article vol. 16, no. 9, pp. 1606-1612, SEP 19981998.

[4] X. Shu, L. Zhang, and I. Bennion, "Sensitivity characteristics of long-period fiber gratings," (in English), Journal of Lightwave Technology, Article vol. 20, no. 2, pp. 255-266, FEB 2002 2002, Art. no. PII S0733-8724(02)00496-6.

[5] G. Rego, J. Santos, and H. Salgado, "Refractive index measurement with long-period gratings arc-induced in pure-silica-core fibres," (in English), Optics Communications, Article vol. 259, no. 2, pp. 598602, MAR 1520062006.

[6] S. James and R. Tatam, "Optical fibre long-period grating sensors: Characteristics and application," (in English), Measurement Science \& Technology, Review vol. 14, no. 5, pp. R49-R61, MAY 2003 2003, Art. no. PII S0957-0233(03)55184-0.

[7] A. Vengsarkar, P. Lemaire, J. Judkins, V. Bhatia, T. Erdogan, and J. Sipe, "Long-period fiber gratings as band-rejection filters," (in English), Journal of Lightwave Technology, Article vol. 14, no. 1, pp. 5865, JAN 19961996.

[8] A. M. Vengsarkar, P. J. Lemaire, J. B. Judkins, V. Bhatia, T. Erdogan, and J. E. Sipe, "Long-period fiber gratings as band-rejection filters," Journal of lightwave technology, vol. 14, no. 1, pp. 58-65, 1996.

[9] G. Rego, "A review of refractometric sensors based on long period fibre gratings," ScientificWorldJournal, vol. 2013, p. 913418, 2013.

[10] S. Khaliq, S. James, and R. Tatam, "Enhanced sensitivity fibre optic long period grating temperature sensor," (in English), Measurement Science \& Technology, Article vol. 13, no. 5, pp. 792-795, MAY 2002 2002, Art. no. PII S0957-0233(02)338177.

[11] E. Simoes, I. Abe, J. Oliveira, O. Frazao, P. Caldas, and J. Pinto, "Characterization of optical fiber long period grating refractometer with nanocoating," (in English), Sensors and Actuators B-Chemical, Article vol. 153, no. 2, pp. 335-339, APR 2020112011.

[12] I. D. Villar, I. R. Matías, F. J. Arregui, and P. Lalanne, "Optimization of sensitivity in Long Period Fiber Gratings with overlay deposition," Optics Express, vol. 13, no. 1, pp. 56-69, 2005/01/10 2005.

[13] I. Del Villar, C. R. Zamarreño, M. Hernaez, F. J. Arregui, and I. R. Matias, "Resonances in coated long period fiber gratings and cladding removed multimode optical fibers: a comparative study," Optics Express, vol. 18, no. 19, pp. 20183-20189, 2010/09/13 2010.

[14] I. D. Villar, I. R. Matias, F. J. Arregui, and M. Achaerandio, "Nanodeposition of materials with complex refractive index in long-period fiber gratings," Journal of lightwave technology, vol. 23, no. 12 , p. 4192, 2005

[15] N. Rees, S. James, R. Tatam, and G. Ashwell, "Optical fiber long-period gratings with LangmuirBlodgett thin-film overlays," (in English), Optics Letters, Article vol. 27, no. 9, pp. 686-688, MAY 1 20022002.

[16] L. Coelho, J. L. Santos, D. Viegas, and J. M. M. M. d. Almeida, "Fabrication and Characterization of Metal Oxide-Coated Long-Period Fiber Gratings," Journal of Lightwave Technology, vol. 34, no. 10, pp. 2533-2539, 2016.

[17] V. Bhatia and A. M. Vengsarkar, "Optical fiber longperiod grating sensors," Optics Letters, vol. 21, no. 9, pp. 692-694, May 11996.

[18] B. H. Lee et al., "Interferometric fiber optic sensors," Sensors (Basel), vol. 12, no. 3, pp. 2467-86, 2012.

[19] H. Patrick, G. Williams, A. Kersey, J. Pedrazzani, and A. Vengsarkar, "Hybrid fiber Bragg grating/long period fiber grating sensor for strain/temperature discrimination," IEEE Photonics Technology Letters, vol. 8, no. 9, pp. 1223-1225, 1996.

[20] Y. Liu, J. Williams, and I. Bennion, "Optical bend sensor based on measurement of resonance mode splitting of long-period fiber grating," IEEE Photonics Technology Letters, vol. 12, no. 5, pp. 531533, 2000.

[21] G. Bai-Ou, T. Hwa-Yaw, T. Xiao-Ming, and D. $\mathrm{Xiao}-\mathrm{Yi}$, "Simultaneous strain and temperature measurement using a superstructure fiber Bragg grating," IEEE Photonics Technology Letters, vol. 12, no. 6, pp. 675-677, 2000.

[22] L. Coelho et al., "Aptamer-based fiber sensor for thrombin detection," Journal of Biomedical Optics, vol. 21, no. 8, pp. 087005-087005, 2016.

[23] L. Coelho, D. Viegas, J. L. Santos, and J. M. M. M. de Almeida, "Optical sensor based on hybrid FBG/titanium dioxide coated LPFG for monitoring organic solvents in edible oils," Talanta, vol. 148, pp. 170-176, 2/1/ 2016.

[24] L. Coelho, D. Viegas, J. Santos, and J. M. M. M. de Almeida, "Zinc oxide coated optical fiber long period gratings for sensing of volatile organic compounds," in SPIE Photonics Europe, 2016, pp. 989918989918-11: International Society for Optics and Photonics.

[25] L. Coelho, D. Viegas, J. L. Santos, and J. M. M. M. de Almeida, "Detection of Extra Virgin Olive Oil Thermal Deterioration Using a Long Period Fibre Grating Sensor Coated with Titanium Dioxide," Food and Bioprocess Technology, journal article vol. 8, no. 6, pp. 1211-1217, 2015.

[26] L. Coelho, J. A. Moreira, P. B. Tavares, J. L. Santos, D. Viegas, and J. M. M. M. de Almeida, "Monitoring of oxidation phases of copper thin films using long period fiber gratings," Sensors and Actuators A: Physical, vol. 253, pp. 69-74, 1/1/ 2017. 
